\title{
Provarieties and observable subgroups of pro-affine algebraic groups
}

\author{
Andy R. Magid \\ Department of Mathematics \\ University of Oklahoma \\ Norman OK 73019
}

\author{
Nazih Nahlus \\ Department of Mathematics \\ American University of Beirut \\ Beirut, Lebanon
}

May 7, 2001

\begin{abstract}
We define a closed subgroup $H$ of a pro-affine algebraic group $G$ to be observable if every (finite dimensional) rational $H$ module is an $H$ submodule of a rational $G$ module. We show the equivalence to observability of a number of other conditions on the pair $G, H$, including the condition that the provariety $G / H$ be pro-quasi-affine. To treat this latter, we also include results on the foundations of provarieties.
\end{abstract}

\section{Introduction}

Let $K$ be an algebraically closed field, let $G$ be an affine algebraic group over $K$ and let $H$ be a (Zariski) closed subgroup of $G$. Let $V$ be a rational $H$ module. Whether $V$ occurs as an $H$-submodule of a rational $G$ module $W$ is a natural question and often an important technical step in applications. When every $V$ so occurs, $H$ is termed an observable subgroup of $G$. Observable subgroups were investigated by Bialynicki-Birula, Hochschild, and Mostow [1]; and by Grosshans [2],[3]; we recall and summarize their main result below (Remark (1)). (Some recent investigations of observability are found in [9] and [13].) Along with the various algebraic conditions equivalent to observability, one also has the geometric condition that $H$ is observable in $G$ if and only if $G / H$ is a quasi-affine variety.

Our purpose in this work is to investigate the condition of observability for a closed subgroup $H$ of a pro-affine algebraic group $G$.

The theory of pro-affine algebraic groups over $K$ arises from the the correspondence between (commutative) Hopf algebras over $K$ and their groups of $K$ points. The keys in the correspondence between Hopf algebras and pro-affine algebraic groups are the facts that inclusions of Hopf algebras always give rise to surjections on $K$ points, that Hopf algebras are rings of $K$ valued functions on their $K$ points, and that surjections of Hopf algebras (or any commutative $K$ algebras) give rise to inclusions of $K$ points. 
For a pro-affine algebraic group $G$ over $K$ with coordinate ring $K[G]$, the identification of $G$ with the set $\operatorname{Alg}_{K}(K[G], K)$ of $K$ algebra homomorphisms from $K[G]$ to $K$, or $K$ points of $K[G]$, also identifies $G$ set theoretically as the inverse limit of the affine algebraic groups $G_{i}=\operatorname{Alg}_{k}\left(A_{i}, k\right)$, where $A_{i}$ ranges over the finitely generated $K$ Hopf subalgebras of $K[G] . K[G]$ is the directed union of the $A_{i}$ so that the set of $K$ points of $K[G]$ is the inverse limit of the set of $K$ points of the $A_{i}$.

The theory of pro-affine algebraic groups, their closed subgroups, and their coordinate rings and rational modules, is sufficient to obtain the various algebraic equivalent conditions to observability, which we do in Section (2) below.

It is the identification $G=\lim G_{i}$ which gives $G$ its pro-affine structure. We note, however, that this structure as just presented does not arise from viewing $G$ as a group in a category of pro-affine varieties, although such an approach is possible. However the construction of a category of pro-affine varieties, or more generally of provarieties, is much more delicate than the correspondence between $K$ Hopf algebras and pro-affine algebra groups. Unlike the situation with the latter, there are reduced $K$ algebras with no $K$ points, and hence which do not correspond in any meaningful way to pro-affine varieties (the statement in [12, p. 190] asserting such a correspondence is in error). For most purposes, in fact, it seems best to avoid the problem of the foundational problems of provarieties when dealing with pro-affine algebraic groups. For the investigations in this paper, however, the issue is unavoidable, since we also want to obtain a geometric condition on $G / H$ for $H$ being observable in the pro-affine group $G$, and $G / H$ is not, in general, either an algebraic variety or a pro-affine algebraic group. In Section (3) below we provide our definition of provariety, which we then use to obtain our geometric condition equivalent to observability.

Notation and Conventions. We retain the notation $K$ for our base field throughout. For a pro-affine algebraic group $G$ over $K$ we use $K[G]$ for its coordinate ring. If $H$ is any (not necessarily closed) subgroup of $G$ then $H^{\prime}=K[G]^{H}$ and if $S$ is any subset of a $G$ module then $S^{\prime}=\{g \in G \mid g s=s \quad \forall s \in S\}$. If $A$ is an integral domain contained in a field $E$ we use q.f.(A) to denote the quotient field of $A$ inside $E$.

In the above notations, our main theorem is as follows:

Main Theorem. Let $G$ be a connected pro-affine algebraic group over $K$ and let $H$ be an algebraic subgroup. Then the following are equivalent:

1. $H$ is observable in $G$.

2. For every 1-dimensional rational $H$ module that is an $H$ submodule of some finite dimensional rational $G$ module, the dual $H$ module is also a submodule of a finite dimensional rational $G$ module. 
3. q.f. $\left(K[G]^{H}\right)=K(G)^{H}$.

4. $H=H^{\prime \prime}$.

5. $H=S^{\prime}$ for some subset $S$ of some rational $G$ module.

6. $K[G]^{H}$ separates the elements of $G / H$.

7. $G / H$ is a quasi-affine provariety. More precisely, there is a bijective morphism of provarieties from $G / H$ (with its natural provariety structure) to a provariety $W=\lim _{\longleftarrow} W_{i}$ where each $W_{i}$ is a quasi-affine algebraic variety

We prove the equivalence of (1) to (6) in Section (2) (Theorem (3)) and add the equivalence of (6) and (7) in Section (3) (Theorem (5)). We also define and characterize quasi-affine provarieties in Section (3).

Remark 1. For the case when $G$ is affine, the equivalent conditions of our main theorem are due to Bialynicki-Birula, Hochschild, and Mostow [1]; and Grosshans [2],[3].

For many of the equivalences, our proofs involve a reduction from the proaffine to the affine case, and then an application of the results of [1] and [2],[3].

\section{Observability}

For general results on pro-affine algebraic groups we refer to [4], [5], [6], and [14].

Let $G$ be a pro-affine algebraic group over $K$ and let $\left\{A_{i} \mid i \in I\right\}$ be the set of finitely generated Hopf subalgebras of $K[G]$. For each $i \in I$ let $G_{i}$ be the affine algebraic group with $K\left[G_{i}\right]=A_{i}$. Then $K[G]=\cup A_{i}$ and hence $G=\lim G_{i}$. We refer to this description of $G$ as the standard limit for $G$ [11, p. 77]. Note that the transition maps $G_{i} \rightarrow G_{j}$ in the standard limit are all surjective.

Remark 2. Let $G=\varliminf_{\varlimsup} G_{i}$ be the standard limit for $G$. Then:

1. Each projection $p_{i}: G \rightarrow G_{i}$ is surjective. Let $K_{i}=\operatorname{Ker}\left(p_{i}\right)$

2. Let $\left\{X_{i} \mid i \in I\right\}$ be a sub inverse system of $\left\{G_{i} \mid i \in I\right\}$ where each $X_{i}$ is a left coset of an algebraic subgroup of $G_{i}$. Then $\varliminf_{i} X_{i}$ is non-empty.

3. If $f: G \rightarrow P$ is a surjective morphism onto an affine algebraic group $P$, then for some $i, f$ induces an isomorphism $G_{i} \rightarrow P$. 
4. The Zariski topology on $G$ is the inverse limit topology.

Proof. Both (1) and (2) follow from [4]. Since $f^{*} K[P]$ is a finitely generated Hopf subalgebra of $K[G]$, it coincides with some $A_{i}$, which proves (3). And (4) follows from Proposition (5) of Section (3)

Notation 1. Let $p_{i}: G \rightarrow G_{i}$ be as in Remark (2) (1). Let $X$ be any subset of $G$. Then $X_{i}$ denotes $p_{i}(X)$. (Note that the two meanings of $G_{i}$ conincide since $p_{i}$ is onto.)

Lemma 1. Let $G=\lim _{i} G_{i}$ be the standard limit for $G$. Then:

1. If $H$ is an algebraic subgroup of $G$ then $H=\lim H_{i}$. Moreover if $H=$

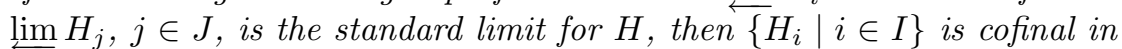
$\left.\overleftarrow{\{H}_{j} \mid j \in J\right\}$.

2. Let $\left\{H_{i}\right\}$ be a sub inverse system of $\left\{G_{i}\right\}$ where each $H_{i}$ is an algebraic subgroup of $G_{i}$. Then $H=\lim _{i} H_{i}$ is an algebraic subgroup of $G$. Moreover, if the transition maps in the system $\left\{H_{i}\right\}$ are surjective, $p_{i} \mid H$ is surjective.

3. If $H$ is an algebraic subgroup of $G$ then $K\left[G_{i}\right]^{H}=K\left[G_{i}\right]^{H_{i}}$. Moreover, if $G$ is connected, then $K\left(G_{i}\right)^{H}=K\left(G_{i}\right)^{H_{i}}$.

Proof. By definition, the morphism $H \rightarrow H_{i}$ is surjective, and of course $H_{i}$ is an algebraic subgroup of $G_{i}$. We identify $K\left[H_{i}\right]$ with its image in $K[H]$. Let $\rho: K[G] \rightarrow K[H]$ come from restriction, so $\rho^{*}\left(K\left[G_{i}\right]\right)=K\left[H_{i}\right]$. Since $K[G]=\cup K\left[G_{i}\right]$, we can apply $\rho^{*}$ to see that $K[H]=\cup K\left[H_{i}\right]$, and this union is directed. It follows that $H=\lim H_{i}$. It also follows that every finite subset of $K[H]$ lies in some $K\left[H_{i}\right]$. So if $H=\varliminf_{\lim } H_{j}$ is the standard limit for $H$, each $K\left[H_{j}\right]$ lies in some $K\left[H_{i}\right]$. Hence (1).

Since $\left\{H_{i}\right\}$ is a sub inverse system of $\left\{G_{i}\right\}, \underset{\lim }{\longleftarrow} H_{i}=\cap p_{i}^{-1} H_{i}$, and each $p_{i}^{-1}\left(H_{i}\right)$ is closed in $G$, so their intersection is as well, which makes the inverse limit a closed, hence algebraic, subgroup of $G$. If the transition maps are surjective, so are the projections by [4, Prop. 2.8, p. 505]. Hence (2).

As a subalgebra of $K[G], K\left[G_{i}\right]$ is identified with $K[G]^{K_{i}}$. Hence $K\left[G_{i}\right]^{H}=$ $K[G]^{K_{i} H}=\left(K[G]^{K_{i}}\right)^{K_{i} H / K_{i}}=K\left[G_{i}\right]^{H_{i}}$. When $G$ is connected, $K\left(G_{i}\right)=$ $K(G)^{K_{i}}$, and then the same identities apply to fields of quotients. Hence (3).

It is a simple consequence of the above that representations of algebraic subgroups of $G$ factor through finite dimensional quotients of $G$, a fact which we now record:

Lemma 2. Let $H$ be an algebraic subgroup of $G$, let $V$ be a finite dimensional rational $H$ module and let $f: H \rightarrow A u t(V)$ be the associated morphism. Let $G=\lim G_{i}, i \in I$, be the standard limit for $G$. Then there is $i \in I$ and a morphism $f_{i}: H_{i} \rightarrow$ Aut $(V)$ such that $f=f_{i} \circ p_{i}$. 
Proof. Since $f(H)$ is an affine algebraic subgroup of $\operatorname{Aut}(V)$, we can apply Lemma (1) (1) and Remark (2) (2).

Lemma (2) allows one to reduce a number of results about observability immediately to the affine case: in the notation of the lemma, if $V$ is an $H_{i}$ submodule of a $G_{i}$ module, this makes it an $H$ submodule of a $G$ module. We record a number of these in the following theorem:

Theorem 1. Let $H$ be an algebraic subgroup of the pro-affine algebraic group $G$.

1. If $H$ is normal in $G$ it is observable in $G$.

2. If $H$ has pro-nilpotent radical (for example, if $H$ is pro-reductive or prounipotent), then $H$ is observable in $G$.

3. If $G$ is pro-solvable then $H$ is observable in $G$.

Proof. As noted, we apply the corresponding results from [1] and [2],[3] for the affine case.

The following topological result, which will be needed for our main theorem, also has independent interest:

Theorem 2. Let $G$ be a pro-affine algebraic group over $K$. Then any family of closed left cosets of $G$ with the finite intersection property has non-empty intersection.

Proof. Let $\left\{S^{t} \mid t \in T\right\}$ be a family of closed left cosets of $G$ with the finite intersection property. Let $G=\lim _{i} G_{i}$ be the standard limit for $G$. Since the projections $p_{i}$ map closed subgroups to algebraic, hence closed, subgroups, for each $i,\left\{p_{i}\left(S^{t}\right)=\left(S^{t}\right)_{i}\right\}$ is a family of closed left cosets, which has the finite intersection property since $\left\{S^{t}\right\}$ does. Let $X_{i}=\cap_{t}\left(S^{t}\right)_{i}$. Since $G_{i}$ is Noetherian, $X_{i}$ is a finite subintersection, and hence non-empty. It follows that $X_{i}$ is itself a left coset of a subgroup of $G_{i}$. It is clear that $\left\{X_{i}\right\}$ is a sub inverse system of $\left\{G_{i}\right\}$, and so by Remark (2) (2) $X=\lim X_{i}$ is non-empty. Let $x \in X$ and let $x_{i}=p_{i}(x)$. For given $t$, we have $x_{i} \in\left(\overleftarrow{S^{t}}\right)_{i}$ for all $i$, and hence $x \in \underline{\lim }\left(S^{t}\right)_{i}$. Since $S^{t}$ is closed in $G$, by Lemma (1) (1), $S^{t}=\varliminf_{(i m}\left(S^{t}\right)_{i}$. Thus $x \in S^{t}$. Since this holds for every $t$, we conclude that $\cap_{t} S^{t} \neq \emptyset$.

As a consequence, we obtain the following analogue of [10, Satz 1]:

Corollary 1. Let $G$ be a proaffine algebraic group, let $H$ be an arbitrary group, and let $f: G \rightarrow H$ be a group homomorphism. Assume that $\operatorname{Ker}(f)$ is closed in G. Let $\left\{A_{t} \mid t \in T\right\}$ be a family of closed left cosets of $G$ and assume that for every pair $s, t \in T$ there is $r \in T$ such that $A_{r} \subseteq A_{s} \cap A_{t}$. then $f\left(\cap A_{t}\right)=\cap f\left(A_{t}\right)$. 
Proof. Let $y \in \cap f\left(A_{t}\right)$. Since $\operatorname{Ker}(f)$ is closed, $f^{-1}(y)$ is a closed left coset. Then the family $\left\{f^{-1}(y) \cap A_{t} \mid t \in T\right\}$ of (non-empty) closed left cosets has the finite intersection property and hence by Theorem (2) their intersection is non-empty. If $z$ belongs to the intersection, then $z \in \cap A_{t}$ and $f(z)=y$, so $y \in f\left(\cap A_{t}\right)$ and thus $\cap f\left(A_{t}\right) \subseteq f\left(\cap A_{y}\right)$. The opposite inclusion is automatic and the result follows.

As noted in Remark (1), one condition equivalent to $H$ observable in $G$ in the affine case is that $H=H^{\prime \prime}$. Because $G$ and $H$ are finite dimensional, this is easily seen to be the same as $H=S^{\prime}$ for some finite subset $S \subset K[G]$. We carry this condition over to the pro-affine case:

Definition 1. Let $H$ be an algebraic subgroup of the pro-affine algebraic group $G$. Then $H$ is super observable in $G$ if there is a finite subset $S \subset K[G]$ such that $H=S^{\prime}=\{g \in G \mid g s=s \quad \forall s \in S\}$.

In this terminology we have:

Lemma 3. Let $H$ be super observable in $G$ and let $G=\lim G_{i}, i \in I$ be the standard limit. Then there is $i_{0} \in I$ such that for all $i \geq i_{0}, \overleftarrow{H}_{i}$ is observable in $G_{i}$. Consequently, $H$ is observable in $G$.

Proof. Let $S \subset K[G]$ be finite such that $H=S^{\prime}$. Choose $i_{0}$ such that $S \subset$ $K\left[G_{i_{0}}\right]$. Let $i \geq i_{0}$. By Lemma (1) (3), $K\left[G_{i}\right]^{H}=K\left[G_{i}\right]^{H_{i}}$, from which it follows that $H_{i}=\left\{g \in G_{i} \mid g s=s \quad \forall s \in S\right\}$. Then by Remark (1), $H_{i}$ is observable in $G_{i}$, so every $H_{i}$ module is an $H_{i}$ submodule of a $G_{i}$ module. Since this is true for every $i \geq i_{0}$, it follows from Lemma (2) that $H$ is observable in $G$.

In final preparation for the proof of the algebraic cases of the main theorem, we record the following facts about closed subgroups of pro-affine groups:

Proposition 1. Let $H$ be an algebraic subgroup of the pro-affine algebraic group $G$ (connected when required). Then:

1. There is a subset $E$ of $K[G]$ such that

$$
H=\{g \in G \mid g s \in K s \quad \forall s \in E\} .
$$

2. $H=q \cdot f \cdot\left(K(G)^{H}\right)^{\prime}$

3. $K(G)^{H}$ separates the elements of $G / H$

Proof. Let $G=\underline{\lim } G_{i}$ be the standard limit for $G$. Select a subset $E_{i}$ of $K\left[G_{i}\right]$ meeting condition (1) for $H_{i}$ in $G_{i}$. Let $E=\cup E_{i}$. It is a straightforward application of Lemma (1) (3) to see that $E$ satisfies (1) for $H$ in $G$. 
The same lemma also noted that $K\left(G_{i}\right)^{H}=K\left(G_{i}\right)^{H_{i}}$. Thus $K(G)^{H}=$ $\cup K\left(G_{i}\right)^{H_{i}}$, and (2) follows from the similar assertion in the affine case.

Finally, (3) follows from Proposition (3) below.

We can now prove the non-geometric parts of our Main Theorem:

Theorem 3. Let $G$ be a connected pro-affine algebraic group over $K$ and let $H$ be an algebraic subgroup. Then the following are equivalent:

1. $H$ is observable in $G$.

2. For every 1-dimensional rational $H$ module that is an $H$ submodule of some finite dimensional rational $G$ module, the dual $H$ module is also a submodule of a finite dimensional rational $G$ module.

3. $q . f \cdot\left(K[G]^{H}\right)=K(G)^{H}$.

4. $H=H^{\prime \prime}$.

5. $H=E^{\prime}$ for some subset $E$ of some rational $G$ module.

6. $K[G]^{H}$ separates the elements of $G / H$.

Proof. The equivalence of (1) and (2) is identical with the affine case $[1$, Thm. $1]$. The proof that (2) implies (3) is also word for word the same as the affine case $\left[1\right.$, Thm. 4.3]. Now assume that q.f. $\left(K[G]^{H}\right)=K(G)^{H}$. Recall that we use $(\cdot)^{\prime}$ to stand for the elements in $G$ which act trivially on $(\cdot)$. We have $\left(K[G]^{H}\right)^{\prime} \subseteq\left(q . f .\left(K[G]^{H}\right)\right)^{\prime}$ and under our assumption this latter is $\left(K(G)^{H}\right)^{\prime}$. By Proposition (1) $(2),\left(K(G)^{H}\right)^{\prime}=H$, so $\left(K[G]^{H}\right)^{\prime} \subseteq H$. The reverse inclusion is trivial and hence we conclude $H^{\prime \prime}=H$. Thus (3) implies (4). That (4) implies (5) is trivial. Now assume that $H=E^{\prime}$, where $E$ is a subset of some rational $G$ module $V$. Let $V^{o}$ be the $K$ dual of $V$, as a rational $G$ module. For $f \in V^{o}$ and $e \in E$ let $f / e \in K[G]$ be defined by $(f / e)(g)=f(g e)$. Let $V_{E}=\left\{f / e \mid f \in V^{o}, e \in E\right\} \subset K[G]$. One checks that in fact $V_{E} \subset K[G]^{H}$. We regard $K[G]^{H}$ as functions on $G / H$. Suppose that $x H$ and $y H$ are in $G / H$ and suppose that $(f / e)(x H)=(f / e)(y H)$ for every $f / e \in V_{E}$. Then $x e=y e$ for all $e \in E$, so that $x^{-1} y \in E^{\prime}=H$. Thus $x H=y H$. It follows that $V_{E}$, and hence $K[G]^{H}$, separates $G / H$. Thus (5) implies (6). Now suppose that $K[G]^{H}$ separates $G / H$, and let $g \in H^{\prime \prime}=\left(K[G]^{H}\right)^{\prime}$. For $f \in K[G]^{H}$ and $h \in H$ we have $f(g h)=(h \cdot f)(g)=f(g)=(g \cdot f)\left(1_{G}\right)=f\left(1_{G}\right)$, so that $f(g H)=f(H)$. Since by assumption $K[G]^{H}$ separates $G / H$, we have that $g H=H$, so that $g \in H$. Thus $H^{\prime \prime} \subseteq H$. The reverse inclusion is trivial and hence $H=H^{\prime \prime}$. Thus (6) implies (4). To complete the equivalences, we assume that $H=H^{\prime \prime}$. Let $V$ be 
a finite dimensional rational $H$ module and $f: H \rightarrow \operatorname{Aut}(V)$ the corresponding representation. Let $G=\lim G_{i}, i \in I$, be the standard limit for $G$. By Lemma (2), there is $i \in I$ and a morphism $f_{i}: H_{i} \rightarrow \operatorname{Aut}(V)$ such that $f=f_{i} \circ p_{i}$. Let $\mathcal{S}=\left\{S^{t} \mid t \in T\right\}$ be the set of all the subgroups of $G$ of the form $E^{\prime}$ where $E \subset H^{\prime}=K[G]^{H}$ is finite. Note that $H=\cap S^{t}$, and that each $S^{t}$ is a super observable subgroup of $G$ in the sense of Definition (1). $\mathcal{S}$ is closed under finite intersections, and it follows from Corollary (1) that $p_{i}\left(\cap S^{t}\right)=\cap p_{i}\left(S^{t}\right)$, hence $H_{i}=p_{i}(H)=p_{i}\left(\cap S^{t}\right)=\cap_{t}\left(S^{t}\right)_{i}$. Because $G_{i}$ is Noetherian and $\mathcal{S}$ is closed under finite intersections, for fixed $i$ there is some $r \in T$ with $\cap_{t}\left(S^{t}\right)_{i}=\left(S^{r}\right)_{i}$. Thus $H_{i}=\left(S^{r}\right)_{i}$. Because $S^{r}$ is super observable, by Lemma (3) there is $i_{0} \in I$ such that for all $j \geq i_{0},\left(S^{r}\right)_{j}$ is observable in $G_{j}$. We can take $j \geq i$. We regard the representation $f_{i}$ of $H_{i}$ as a representation of $\left(S^{r}\right)_{i}$ and hence as a representation of $\left(S^{r}\right)_{j}$. As such, $V$ is an $\left(S^{r}\right)_{j}$ submodule of a $G_{j}$ module $W$. We regard $W$ as a $G$ module via $p_{j}$, and then $V$ becomes as $H$ submodule of $W$. Hence (4) implies (1).

\section{Provarieties}

As shown above, a closed subgroup $H$ of the pro-affine algebraic groups $G$ is proaffine, and in fact $H$ is the inverse limit of subgroups $H_{i}$ of the groups $G_{i}$ defined above. It follows easily that $G / H$ can be regarded as the (set theoretic) inverse limit of the inverse system of varieties $G_{i} / H_{i}$. We need to use this inverse limit structure to regard $G / H$ as a provariety, and to make some general geometric comments about $G / H$ based on this provariety structure.

We remark that provarieties appear in the literature in various contexts, including those that arise from inverse limits of algebraic groups. In addition to Peterson [12] who considers pro-affine algebraic groups, Kovacic [7] considers inverse limits of non affine algebraic groups, and Kowalski and Pillay [8] consider groups in the category of pro-algebraic varieties. Kovacic only considers groups which will not cover our case $G / H$ when $H$ is not normal. For Kowalski and Pillay, a provariety is an inverse system, not the inverse limit, which we want to use (and they restrict their inverse systems to countable ones). Therefore, we propose the following definition of provarieties:

Definition 2. Let $\left(X_{\alpha}, p_{\alpha, \beta}\right), \alpha \in \mathcal{A}, p_{\beta, \alpha}: X_{\beta} \rightarrow X_{\alpha}$ if $\beta \geq \alpha$ be an inverse system of irreducible algebraic varieties over $K$ with directed index set $\mathcal{A}$ and dominant transition maps. We denote the function field of $X_{\alpha}$ by $K\left(X_{\alpha}\right)$ and let $p_{\alpha, \beta}^{*}: K\left(X_{\alpha}\right) \rightarrow K\left(X_{\beta}\right)$ if $\beta \geq \alpha$. We let $K(X)$ denote $\lim _{\longrightarrow} K\left(X_{\alpha}\right)$ and refer to it as the field of rational functions on $X$. We let $p_{\alpha}^{*} \overrightarrow{\text { denote the canonical }}$ injection $K\left(X_{\alpha}\right) \rightarrow K(X)$. Then $X=\lim X_{\alpha}$ is defined to be the ringed space $\left(X, \mathcal{O}_{X}\right)$ defined as follows:

(a) $X$ is the topological inverse limit of the spaces $X_{\alpha}$. Let $p_{\alpha}: X \rightarrow X_{\alpha}$ denote the canonical projection. Basic open subsets of $X$ are of the form $U=p_{\alpha}^{-1}\left(U_{\alpha}\right)$, where $U_{\alpha} \subseteq X_{\alpha}$ is open. 
(b) Let $f \in K(X)$ and $x \in X$. Then $f$ is said to be defined at $x$ if there is a basic open set $U=p_{\alpha}^{-1}\left(U_{\alpha}\right)$ such that $x \in U$ and $f=p_{\alpha}^{*}\left(f_{\alpha}\right)$ for some $f_{\alpha} \in \Gamma\left(U_{\alpha}, \mathcal{O}_{X_{\alpha}}\right)$. We call $U$ a defining neighborhood of $f$ at $x$. Note that if $U$ is a defining neighborhood for $f$, then $f$ is defined at every point of $U$.

(c) Define the presheaf $\mathcal{O}_{X}$ as follows: for $U \subset X$, let $\Gamma\left(U, \mathcal{O}_{X}\right)=\{f \in K(X) \mid$ $f$ is defined at $x \forall x \in U\}$. For the empty set, we define $\Gamma\left(\emptyset, \mathcal{O}_{X}\right)=0$. Note that, by definition, $\Gamma\left(U, \mathcal{O}_{X}\right) \subseteq K(X)$. If $U \subseteq V$ is an inclusion of open sets, $\Gamma\left(V, \mathcal{O}_{X}\right) \rightarrow \Gamma\left(U, \mathcal{O}_{X}\right)$ denotes the inclusion for $U$ nonempty, and the zero map otherwise.

We then have the following consequences of Definition (2)

Proposition 2. Let $X=\varliminf_{\varlimsup} X_{\alpha}$ be a provariety. The presheaf $\mathcal{O}_{X}$ is a sheaf of $K$ algebras and for $x \in \overleftarrow{X}$ the stalk $\mathcal{O}_{X, x}$ is $\{f \in K(X) \mid f$ is defined at $x\}$.

Proof. First suppose that $f \in K(X)$ is defined at $x$ and let $U=p_{\alpha}^{-1}\left(U_{\alpha}\right)$ be a basic open set such that $x \in U$ and $f=p_{\alpha}^{*}\left(f_{\alpha}\right)$ for some $f_{\alpha} \in \Gamma\left(U_{\alpha}, \mathcal{O}_{X_{\alpha}}\right)$. Note that if $U^{\prime}=p_{\beta}^{-1}\left(U_{\beta}\right)$ is any basic open set with $x \in U^{\prime}$ and if $\gamma \geq \alpha, \beta$ then for $U_{\gamma}=p_{\gamma, \beta}^{-1}\left(U_{\beta}\right) \cap p_{\gamma, \alpha}^{-1}\left(U_{\alpha}\right), U^{\prime \prime}=p_{\gamma}^{-1}\left(U_{\gamma}\right)$, and $f_{\gamma}=p_{\gamma, \alpha}^{*}\left(f_{\alpha}\right)$, we have $x \in U^{\prime \prime} \subseteq U_{\alpha} \cap U_{\beta}$ and $f=p_{\gamma}^{*}\left(f_{\gamma}\right)$. It follows that if $x$ is defined at $x$ then any open set containing $x$ contains a defining neighborhood of $f$. It further follows that $f$ is determined, as an element of $K(X)$, by any defining neighborhood of any point of definition of $f$. Finally, this also implies that the rational functions defined at $x$ form a $K$ subalgebra of $K(X)$.

Now let $U \subseteq X$ be open and let $\left\{U^{a} \mid a \in A\right\}$ be a cover of $U$ by nonempty basic open sets $U^{a}=p_{\alpha(a)}^{-1}\left(U_{\alpha(a)}\right)$. We need to show that the sequence

$$
\Gamma\left(U, \mathcal{O}_{X}\right) \rightarrow \prod_{a \in A} \Gamma\left(U^{a}, \mathcal{O}_{X}\right) \rightrightarrows \prod_{a, b \in A} \Gamma\left(U^{a} \cap U^{b}, \mathcal{O}_{X}\right)
$$

is a coequalizer. The leftmost map is an injection, in fact for any $a \in A$ the map $\Gamma\left(U, \mathcal{O}_{X}\right) \rightarrow \Gamma\left(U^{a}, \mathcal{O}_{X}\right)$ is an injection. So suppose $\left(f^{a}\right)_{a \in A}$ belongs to the middle product and restricts to the same element of the rightmost product under both maps. It follows that as elements of $K(X), f^{a}=f^{b}$ for all $a, b \in A$. Fix some $b \in A$, and let $f=f^{b}$ as an element of $K(X)$. If $x \in U$, and $x \in U^{a}$, then $f=f^{a}$ is defined at $x$, and it follows that $f \in \Gamma\left(U, \mathcal{O}_{X}\right)$ and that $f$ has image $\left(f^{a}\right)_{a}$ under the leftmost map. Thus $\mathcal{O}_{X}$ is a sheaf.

Finally, it is clear that if $x \in X$ then the stalk $\mathcal{O}_{X, x}$ consists of functions defined at $x$, since it is the direct limit (union) of subalgebras of $K(X)$ of functions defined at $x$. Conversely, if $f \in K(X)$ is defined at $x$, and $U$ is any defining set for $f$ at $x$, then $f \in \Gamma\left(U, \mathcal{O}_{X}\right)$, at the latter algebra of sections in contained in $\mathcal{O}_{X, x}$.

We record the observation that the sections of $\mathcal{O}_{X}$ in Proposition (2) over basic open sets have a natural direct limit structure, and hence that stalks are direct limits. 
Lemma 4. Let $X=\lim X_{\alpha}$ be a provariety and let $U=p_{\alpha}^{-1}\left(U_{\alpha}\right)$, where $U_{\alpha} \subseteq$ $X_{\alpha}$ is open, be a basic open set. Then $p_{\alpha}^{*}\left(\Gamma\left(U_{\alpha}, \mathcal{O}_{U_{\alpha}}\right) \subseteq \Gamma\left(U, \mathcal{O}_{X}\right)\right.$. Moreover if $f \in \Gamma\left(U, \mathcal{O}_{X}\right)$, then there is $\beta \geq \alpha$, an open $U_{\beta} \subseteq p_{\beta, \alpha}^{-1}\left(U_{\alpha}\right)$ and an $f_{\beta} \in$ $\Gamma\left(U_{\beta}, \mathcal{O}_{U_{\beta}}\right)$ such that $f=p_{\beta}^{*}\left(f_{\beta}\right)$ in $K(X)$. In particular, for $x \in X$

$$
\mathcal{O}_{X, x}=\underline{\lim _{1}} p_{\alpha}^{*}\left(\mathcal{O}_{X_{\alpha}, p_{\alpha}(x)}\right) \text {. }
$$

Under some stronger hypotheses on the inverse system $\left(X_{\alpha}, p_{\alpha, \beta}\right)$ we have the following strengthening of Lemma (4):

Lemma 5. Assume that in the inverse system $\left(X_{\alpha}, p_{\alpha, \beta}\right), \alpha \in \mathcal{A}, p_{\beta, \alpha}: X_{\beta} \rightarrow$ $X_{\alpha}$, for $\beta \geq \alpha$ the transition maps are surjective and satisfy

$$
\mathcal{O}_{X_{\beta}, x_{\beta}} \cap p_{\beta, \alpha}^{*}\left(K\left(X_{\alpha}\right)\right)=p_{\beta, \alpha}^{*}\left(\mathcal{O}_{X_{\alpha}, x_{\alpha}}\right) \text { where } p_{\beta, \alpha}^{*}\left(x_{\beta}\right)=x_{\alpha}
$$

Let $X=\lim _{\alpha} X_{\alpha}$ and suppose that the maps $X \rightarrow X_{\alpha}$ are all surjective. Let $U=p_{\alpha}^{-1}\left(U_{\alpha}\right) \subseteq X$ be basic open. Then

$$
\Gamma\left(U, \mathcal{O}_{X}\right)=\varliminf_{\beta \geq \alpha} p_{\beta}^{*}\left(\Gamma\left(p_{\beta, \alpha}^{-1}\left(U_{\alpha}\right), \mathcal{O}_{X_{\beta}}\right)\right)
$$

and in particular, $\Gamma\left(U, \mathcal{O}_{X}\right) \cap p_{\alpha}^{*}\left(K\left(X_{\alpha}\right)\right)=p_{\alpha}^{*}\left(\Gamma\left(p_{\alpha}(U), \mathcal{O}_{X_{\alpha}}\right)\right)$.

Proof. It is clear from the definition of $\mathcal{O}_{X}$ that the direct limit is contained in $\Gamma\left(U, \mathcal{O}_{X}\right)$. Let $f \in \Gamma\left(U, \mathcal{O}_{X}\right)$. By Lemma (4), for each $x \in U$ there is $\beta(x)=\beta \geq \alpha$ such that $f=p_{\beta}^{*}\left(f_{\beta}\right) \in p_{\beta}^{*}\left(\mathcal{O}_{X_{\beta}, p_{\beta}(x)}\right)$. Fix one such $\beta$; without loss of generality we may assume $\beta=\alpha$. Then for any $x \in U$ and for $\beta=$ $\beta(x), x_{\beta}=p_{\beta}(x)$, and $x_{\alpha}=p_{\alpha}(x)$ we have $p_{\beta, \alpha}^{*}\left(f_{\alpha}\right)=f_{\beta} \in \mathcal{O}_{X_{\beta}, x_{\beta}}$, and by assumption this implies that $f_{\beta} \in p_{\beta, \alpha}^{*}\left(\mathcal{O}_{X_{\alpha}, x_{\alpha}}\right)$. But then $f_{\alpha} \in \mathcal{O}_{X_{\alpha}, x_{\alpha}}$. This holds for every $x \in U$, and hence $f_{\alpha} \in p_{\alpha}^{*}\left(\Gamma\left(U_{\alpha}, \mathcal{O}_{X_{\alpha}}\right)\right)$, as desired.

Note that for this argument it is sufficient simply to have $f=p_{\alpha}^{*}\left(f_{\alpha}\right)$ : for then $f_{\alpha}$ is defined at $x_{\alpha}$ for at some $x_{\alpha} \in U_{\alpha}$, and we can take $x \in p_{\alpha}^{-1}\left(x_{\alpha}\right)$. The final assertion of the lemma follows.

The conclusion of Lemma (5) can be informally phrased as follows: under the hypotheses of the lemma, if a section of $\mathcal{O}_{X}$ over a basic open set $U$ comes from a rational function on $p_{\alpha}(U)$, then it comes from a section of $\mathcal{O}_{X_{\alpha}}$ over $p_{\alpha}(U)$.

We refer below to the hypotheses of Lemma (5) as the transition map conditions. The conditions will be satisfied when the inverse system of $X_{\alpha}$ 's arises from a pro-affine algebraic group $G=\lim _{G_{\alpha}}$ and a pro-affine algebraic subgroup $H$ taking $X_{\alpha}=G_{\alpha} / H_{\alpha}$, where $H_{\alpha}=p_{\alpha}(H)$.

The following proposition analyzes when the global sections of a provariety separates points.

Proposition 3. Let $X=\lim _{X_{\alpha}}$ be a provariety and suppose the inverse system $\left(X_{\alpha}, p_{\alpha, \beta}\right), \alpha \in \mathcal{A}, p_{\beta, \alpha}: X_{\beta} \rightarrow X_{\alpha}$, for $\beta \geq \alpha$ satisfies all the transition conditions of Lemma (5). Assume that $\Gamma\left(X, \mathcal{O}_{X}\right)$ separates the points of $X$. 
Then if $v_{\alpha} \neq w_{\alpha}$ are points of $X_{\alpha}(\alpha \in \mathcal{A})$, there is $\beta \geq \alpha$ and points $v_{\beta}, w_{\beta}$ of $X_{\beta}$ with $p_{\beta, \alpha}\left(v_{\beta}\right)=v_{\alpha}, p_{\beta, \alpha}\left(w_{\beta}\right)=w_{\alpha}$ and $f_{\beta} \in \Gamma\left(X_{\beta}, \mathcal{O}_{X_{\beta}}\right)$ such that $f_{\beta}\left(v_{\beta}\right) \neq f_{\beta}\left(w_{\beta}\right)$. Conversely, if for each $\alpha$ and each pair of points in $X_{\alpha}$ there is $\beta \geq \alpha$ and $f_{\beta} \in \Gamma\left(X_{\beta}, \mathcal{O}_{X_{\beta}}\right)$ separating preimages of those points in $X_{\beta}$, then $\Gamma\left(X, \mathcal{O}_{X}\right)$ separates the points of $X$.

Proof. We begin with $v_{\alpha} \neq w_{\alpha}$, and choose $v, w \in X$ such that $p_{\alpha}(v)=v_{\alpha}$ and $p_{\alpha}(w)=w_{\alpha}$. By assumption, there is $f \in \Gamma\left(X, \mathcal{O}_{X}\right)$ such that $f(v) \neq f(w)$. By Lemma (5), there $f=p_{\beta}^{*}\left(f_{\beta}\right)$ for some $\beta$, which we can assume, without loss of generality, satisfies $\beta \geq \alpha$. Take $v_{\beta}=p_{\beta}(v)$ and $w_{\beta}=p_{\beta}(w)$; then $v_{\beta}, w_{\beta}, f_{\beta}$ meet the conclusions of the proposition. The final statement of the proposition follows from Lemma (5): namely, from

$$
\Gamma\left(X, \mathcal{O}_{X}\right)=\underline{\lim } \Gamma\left(X_{\beta}, O_{X_{\beta}}\right) .
$$

A possible stronger form of Proposition (3), which would assert that a provariety whose points are separated by their global sections is an inverse limit of varieties separated by their global sections (i.e. quasi-affine varieties), is false, as the following example shows:

Example 1. Let $G$ denote $S L_{n}(\mathbb{C})$ and let $B$ denote its Borel subgroup of upper triangular matrices, so $G / B=\mathbb{P}_{1}(\mathbb{C})$. We define an inverse system indexed by the natural numbers $\mathbb{N}$ as follows: take $X_{1}=G$. For $n>1$, let $X_{n}=G^{(n-1)} \times$ $G / B$. Define $p_{n+1, n}$ by projection onto the first $n$ factors followed by the obvious surjection $G^{(n)} \rightarrow G^{(n-1)} \times G / B$. For $n>m$ we define $p_{n, m}$ by composition, and of course $p_{n, n}$ is the identity. The inverse system $\left\{X_{n}, p_{n, m} \mid n \in \mathbb{N}\right\}$ satisfies the transition conditions. Let $X=\varliminf_{(X m} X_{n}$. It is clear from Proposition (3), or by a simple direct argument, that $\Gamma\left(X, \mathcal{O}_{X}\right)$ separates the points of $X$. On the other hand, it is also clear that none of he $X_{i}$ 's are separated by their global sections.

We next want to consider the functorial properties of Definition (2). First, we define morphisms, beginning with a morphism of inverse systems:

Definition 3. Let $\left(X_{\alpha}, p_{\alpha, \beta}\right), \alpha \in \mathcal{A}, p_{\beta, \alpha}: X_{\beta} \rightarrow X_{\alpha}$ if $\beta \geq \alpha$ and $\left(Y_{\gamma}, p_{\gamma, \delta}\right)$, $\gamma \in \mathcal{B}, q_{\delta, \gamma}: Y_{\delta} \rightarrow Y_{\gamma}$ if $\delta \geq \gamma$ be directed inverse systems of irreducible algebraic varieties over $K$ with dominant transition maps. A morphism between the systems is a pair $(\phi, \mathcal{F})$ where $\phi: \mathcal{A} \rightarrow \mathcal{B}$ is an order preserving map with cofinal image and $\mathcal{F}=\left\{f_{\alpha}: X_{\alpha} \rightarrow Y_{\phi(\alpha)} \mid \alpha \in \mathcal{A}\right\}$ is a set of morphisms such that if $\beta \geq \alpha$ then $q_{\phi(\beta), \phi(\alpha)} f_{\beta}=f_{\alpha} p_{\beta, \alpha}$.

For example, if $\left(X_{\alpha}, p_{\alpha, \beta}\right), \alpha \in \mathcal{A}, p_{\beta, \alpha}: X_{\beta} \rightarrow X_{\alpha}$ is an inverse system as in Definition (3) and we fix $\gamma \in \mathcal{A}$, we can let $\mathcal{A}_{0}=\{\gamma\}$ and consider $X_{\gamma}$ as the inverse system over $\mathcal{A}_{0}$ with identity transition map. Then the constant map $\phi: \mathcal{A} \rightarrow \mathcal{A}_{0}$ is order preserving and has cofinal image. Let $\mathcal{F}=\left\{p_{\beta, \gamma} \mid \beta \geq \gamma\right\}$. It follows that $(\phi, \mathcal{F})$ is a morphism from $\left(X_{\alpha}, \alpha \in \mathcal{A}\right)$ to $\left(X_{\gamma}, \gamma \in \mathcal{A}_{0}\right)$. 
A morphism of inverse systems defines a function between their set theoretic inverse limits. In the notation of definition (3), the function $F: X \rightarrow Y$ where $X=\lim X_{\alpha}$ and $Y=\lim Y_{\gamma}$ as sets as defined by the morphism $(\phi, \mathcal{F})$, and given by the conditions $\overleftarrow{f_{\alpha}} p_{\alpha}=q_{\phi(\alpha)} F \forall \alpha \in \mathcal{A}$. Since $\phi(\mathcal{A})$ is cofinal in $\mathcal{B}$ we can replace the latter by the former and assume $\phi$ is surjective. We continue to follow this notation.

Let $y \in Y$, let $g$ be a rational function on $Y$ defined at $y$, and let $V=$ $q_{\gamma}^{-1}\left(V_{\gamma}\right)$, where $V_{\gamma} \subseteq Y_{\gamma}$ is open, be a defining set for $g$ at $y$ with $g=q_{\gamma}^{*}\left(g_{\gamma}\right)$. Suppose $F(x)=y$. Suppose $\gamma=\phi(\alpha)$. Let $U_{\alpha}=f_{\alpha}^{-1}\left(V_{\gamma}\right)$. Then $U_{\alpha} \subseteq X_{\alpha}$ is open and $U=p_{\alpha}^{-1}\left(U_{\alpha}\right)$ is basic open. From $f_{\alpha}$ we have the map $f_{\alpha}^{*} \Gamma\left(V_{\gamma}, \mathcal{O}_{Y_{\gamma}}\right) \rightarrow$ $\Gamma\left(U_{\alpha}, \mathcal{O}_{X_{\alpha}}\right)$. Thus $f=p_{\alpha}^{*} f_{\alpha}^{*} g_{\gamma}$ is, by Lemma (4), a rational function on $X$ defined on $U$. Note that $x \in U$. We define $F_{x}^{*}: \mathcal{O}_{Y, F(x)} \rightarrow \mathcal{O}_{X, x}$ by $g \mapsto f$. (We omit the straight forward verifications that this map is well-defined and that it is a $K$ algebra map.) We can then apply this map to every point of $F^{-1}(V)$ and obtain a map $\Gamma\left(V, \mathcal{O}_{Y}\right) \rightarrow \Gamma\left(F^{-1}(V), \mathcal{O}_{X}\right)$.

We can now show that morphisms of inverse systems can be used to define morphisms of provarieties:

Proposition 4. Let $F: X=\lim _{\longleftarrow} X_{\alpha} \rightarrow Y=\lim Y_{\gamma}$ be a morphism of (the underlying sets of) provarieties arising from a morphism of inverse systems. Then if $V$ is a basic open subset of $Y$ then $F^{-1}(V)$ is a basic open subset of $X$, and hence $F$ is continuous. There is a sheaf morphism $F^{*} \mathcal{O}_{Y} \rightarrow F_{*}\left(\mathcal{O}_{X}\right)$.

Proof. The first assertion was noted in the discussion above. Recall that $F_{*}\left(\mathcal{O}_{X}\right)$ is the sheaf on $Y$ whose section on the open subset $V$ are given by $\Gamma\left(V, F_{*}\left(\mathcal{O}_{X}\right)=\right.$ $\Gamma\left(F^{-1}(V), \mathcal{O}_{X}\right)$; then second assertion was also established above.

Proposition (4) says that the function $F: X \rightarrow Y$ on the underlying sets of the provarieties $X$ and $Y$ gives rise to a map ringed spaces. We extend the notation of the proposition in the usual way: for $V \subseteq Y$ open we have a $K$ algebra map on sections

$$
F_{V}^{*}: \Gamma\left(V, \mathcal{O}_{Y}\right) \rightarrow \Gamma\left(F^{-1}(V), \mathcal{O}_{X}\right)
$$

and for $x \in X$ we have a $K$ algebra map on stalks

$$
F_{x}^{*}: \mathcal{O}_{Y, F(x)} \rightarrow \mathcal{O}_{X, x}
$$

If we combine the above formula for $F_{x}^{*}$ with the final direct limit of Lemma (4), we have the following description of morphisms of provarieties on stalks:

Lemma 6. Let $F: X=\lim _{\alpha} X_{\alpha} \rightarrow Y=\lim Y_{\gamma}$ be a morphism of provarieties arising from the map $(\phi, \overleftarrow{\mathcal{F}})$ of inverse systems. Then for $x \in X$

$$
F_{x}^{*}=\lim _{\longrightarrow, \phi(\alpha)} f_{\alpha} \lim _{\phi(\alpha)}^{*}\left(\mathcal{O}_{Y_{\phi(\alpha}, f_{\alpha}\left(p_{\alpha}(x)\right)}\right) \rightarrow \underline{\lim _{1}} p_{\alpha}^{*}\left(\mathcal{O}_{X_{\alpha}, p_{\alpha}(x)}\right) .
$$


It is a consequence of our definition of provariety and of morphism that if $X=\lim _{\longleftarrow} X_{\alpha}$ is a provariety then the projection $p_{\alpha}: X \rightarrow X_{\alpha}$ is a morphism of provarieties. We also have the following for closed subsets of provarieties:

Let $X=\lim _{\longleftarrow} X_{\alpha}$ be a provariety. Suppose $Y \subseteq X$ satisfies

$$
Y=\cap_{\alpha} p_{\alpha}-1\left(\overline{p_{\alpha}(Y)}\right)
$$

Then the morphism $\lim _{\overline{p_{\alpha}(Y)}}$ has image $Y$. We call such subsets of a provariety saturated, and the content of this remark is that we can regard saturated subsets $Y$ of $X$ as provarieties.

Saturated subsets are clearly closed. We remark that the converse is true as well:

Lemma 7. Let $Y$ be a closed subset of the provariety $X$. Then $Y$ is saturated.

Proof. Suppose $X=\lim X_{\alpha}$ and $Y \subseteq X$ is closed. Let $x \in X--Y$, and let $U=p_{\alpha}^{-1}\left(U_{\alpha}\right)$ be a basic open subset with $x \in U$ and $U \cap Y=\emptyset$. If $z=p_{\alpha}(y) \in U_{\alpha}$ then $y \in U$, so $p_{\alpha}(Y) \cap U_{\alpha}=\emptyset$. Since $U_{\alpha}$ is open, this means that $\overline{p_{\alpha}(Y)} \cap U_{\alpha}$ is empty as well, and thus $p_{\alpha}-1\left(\overline{p_{\alpha}(Y)}\right) \cap U=\emptyset$, so in particular $x \notin p_{\alpha}-1\left(\overline{p_{\alpha}(Y)}\right)$. So $x \notin Y$ implies $x \notin \cap_{\alpha} p_{\alpha}-1\left(\overline{p_{\alpha}(Y)}\right)$, and the contrapositive of this in the assertion of the lemma.

If $\left\{X_{i} \mid i \in \mathcal{I}\right\}$ is any set of algebraic varieties, and $A$ is any subset of $I$, then we let $X_{A}$ denote $\prod_{i \in A} X_{i}$. The set $\mathcal{F}$ of finite subsets of $I$ is partially ordered by inclusion, and directed in this partial order. Set theoretically, we have

$$
\prod_{i \in I} X_{i}=\lim _{\longleftarrow}\left\{X_{F} \mid F \in \mathcal{F}\right\} .
$$

We use this identification to view $\prod X_{i}$ as a provariety. Note that the topology on $\prod X_{i}$ is defined by basic open subsets $U$ of the form $U_{F} \times \prod_{i \notin F} X_{i}$.

Proposition 5. Let $\left(X_{\alpha}, p_{\alpha, \beta}\right)$ be an inverse system of algebraic varieties with inverse limit $X$. Then the canonical image of $X$ in $P=\prod X_{\alpha}$ is saturated and the canonical map $q: X \rightarrow P$ is a morphism of provarieties. Let $q(X)$ be given its provariety structure as a saturated subset. Then $X \rightarrow q(X)$ is an isomorphism of provarieties.

Proof. The image $q(X)$ of $X$ in $P$ is $\left\{\left(x_{\alpha}\right) \mid x_{\alpha}=p_{\beta, \alpha}\left(x_{\beta}\right)\right)$ for $\left.\beta \geq \alpha\right\}$. Thus $q(X)=\cap_{\beta \geq \alpha} G_{\beta, \alpha} \times X_{I-\{\alpha, \beta\}}$ where $\left.\left.G_{\beta, \alpha}=\left\{x_{\beta}, x_{\alpha}\right) \mid x_{\alpha}=p_{\beta, \alpha}\left(x_{\beta}\right)\right)\right\}$ is the graph of $p_{\beta, \alpha}$. These graphs are all closed, of course. Suppose $F \subseteq \mathcal{A}$ is a finite set of indices. The projection $p_{F}(q(X))$ lies inside the subset $X_{F}^{\prime}$ of $X_{F}$ consisting of consistent tuples $\left(x_{a}\right)_{a \in F}$. The same remark on graph closures implies that $X_{F}^{\prime}$ is closed in $X_{F}$, so that $X_{F}^{\prime \prime}=X_{F}^{\prime} \times \prod_{\alpha \notin F}$ is closed in $P$. It is clear that $\overline{p_{F}(q(X))} \subseteq X_{F}^{\prime}$ so that $p_{F}^{-1}\left(\overline{p_{F}(q(X))}\right) \subset X_{F}^{\prime \prime}$, and that $q(X)=\cap_{F} X_{F}^{\prime \prime}$. It follows that $q(X)$ is a saturated subset of $P$.

It remains to identify the provariety structure on $q(X)$ as a saturated subset of $P$ with that of the given provariety structure on $X$. It is trivial that $X \rightarrow q(X)$ 
is a homeomorphism of the underlying topological spaces, and we already know it is a morphism of provarieties. To see that it is an isomorphism of provarieties, it suffices to consider the map on stalks, and then the result follows from the case of finite products by Lemma (6).

We will use Proposition (5) to identify the inverse limit $X$ and the set of consistent tuples $q(X)$.

The combination of Proposition (5) and Lemma (6) provides a description of the stalks of provariety, and in the case of a pro-affine variety, of the coordinate ring:

Corollary 2. Let $\left(X_{\alpha}, p_{\alpha, \beta}\right)$ be an inverse system of algebraic varieties with inverse limit $X$, and let $x=\left(x_{\alpha}\right)$ belong to $X \subseteq \prod X_{\alpha}$. Then:

1. There is a surjection $\otimes_{\alpha} \mathcal{O}_{X_{\alpha}, x_{\alpha}} \rightarrow \mathcal{O}_{X, x}$

2. If the $X_{\alpha}$ are all affine, then there is a surjection $\otimes_{\alpha} \Gamma\left(X_{\alpha}, \mathcal{O}_{X_{\alpha}, x_{\alpha}}\right) \rightarrow$ $\Gamma\left(X, \mathcal{O}_{X, x}\right)$

Of course the surjections in Corollary (2) tacitly assume that $X$ is nonempty. This need not be the case, even when all $X_{\alpha}$ are affine: for example, let $K(x)$ be the field of rational functions over $K$. We can write $K(x)=\underline{\lim } A_{i}$ where $\left\{A_{i} \mid i \in I\right\}$ ranges over the finitely generated $K$ subalgebras of $\overrightarrow{K(x)}$, and form the inverse system of affine varieties $X_{i}$ where $\Gamma\left(X_{i}, \mathcal{O}_{X_{i}}\right)=A_{i}$. Then $\lim X_{i}$ is empty (it corresponds to the $K$ points of $K(x)$ ). In terms of Proposition (5) and its proof, this implies that the closed subsets $X_{F}^{\prime \prime}$, which have the finite intersection property, have empty intersection. In particular, $\prod X_{i}$ is not quasi-compact.

On the other hand, suppose we begin with a set $X$ and an integral domain $A$ of $K$ valued functions on $X$ which separates the points of $X$. Suppose $\left\{A_{\alpha}, \alpha \in\right.$ $\mathcal{A}$ \} is a set of finitely generated $K$ subalgebras of $A$ indexed by the directed set $\mathcal{A}$, and such that $A_{\alpha} \subseteq A_{\beta}$ if $\alpha \leq \beta$. Let $X_{\alpha}=\operatorname{Alg}_{K}\left(A_{\alpha}, K\right)$ be the affine variety with coordinate ring $A_{\alpha}$. Then there is an obvious injection $X \rightarrow \lim _{X_{\alpha}}$. Note that the latter is an inverse limit of affine varieties.

Now suppose $X=\lim Y_{\gamma}$ itself is a provariety over some inverse system $\left(Y_{\gamma}, q_{\gamma, \delta}\right), \gamma \in \mathcal{B}$ and that $A=\Gamma\left(X, \mathcal{O}_{X}\right)$ separates the points of $X$. Suppose further that the finitely generated $K$ algebras $A_{\alpha}$ satisfy the following cofinality condition: for $\gamma \in \mathcal{B} \exists \alpha(\gamma) \in \mathcal{A}$ so that $q_{\gamma}^{*}\left(\Gamma\left(Y_{\gamma}, \mathcal{O}_{Y_{\gamma}}\right)\right) \subseteq A_{\alpha(\gamma)}$. (If each $\Gamma\left(Y_{\gamma}, \mathcal{O}_{Y_{\gamma}}\right)$ is finitely generated, then a cofinal subset of $\mathcal{A}$ always satisfies this.) Then there is a morphism of inverse systems $\left(Y_{\gamma}\right) \rightarrow\left(X_{\alpha}\right)$ and hence a morphism of provarieties $X \rightarrow \lim X_{\alpha}$. This map is injective under the assumption that $A$ separates the points of $X$. We record this conclusion, in slightly different notation, in the following proposition: 


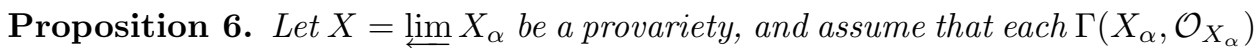
is finitely generated. Suppose that $\Gamma\left(X, \mathcal{O}_{X}\right)$ separates the points of $X$. Then $X$ embeds in a provariety which is an inverse limit of affine varieties. In particular, $X$ embeds in a product of affine varieties.

Proof. The only remaining point is the final assertion, which follows from Proposition (5).

When $A$ is an (integral domain) $K$ algebra whose $K$ points both separate the elements of $A$ and map surjectively to the $K$ points of its finitely generated subalgebras $A_{i}$, the bijection between $\operatorname{Alg}_{K}(A, K)$ and $\varliminf \operatorname{Alg}_{k}\left(A_{i}, k\right)$ identifies the former set theoretically as a provariety $X$, and, by Corollary (2) (2), identifies $A$ and $\Gamma\left(X, \mathcal{O}_{X}\right)$. For the case of pro-affine algebraic groups, we record this in the following proposition.

Proposition 7. Let $G$ be a pro-affine algebraic group. Then the provariety structure on $G$ from definition (2) coincides with the structure of $G$ as a proalgebraic group.

Finally, we want to deal with pro-affine subgroups of pro-affine algebraic groups.

Theorem 4. Let $H$ be a pro-affine subgroup of the pro-algebraic group $G$. Then $G / H$ carries the natural structure of a provariety.

Proof. We use the set of all finitely generated Hopf subalgebras $\left\{A_{\alpha} \mid \alpha \in \mathcal{A}\right\}$ of $K[G]$ to write the latter as a direct limit and let $\left\{G_{\alpha}, p_{\alpha, \beta}\right\}$ be the corresponding inverse system, so $G=\lim _{\alpha} G_{\alpha}$. We let $B_{\alpha}$ be the image of $A_{\alpha}$ in $K[H]$ so that we have an inverse system $\left\{H_{\alpha}, q_{\alpha, \beta}\right\}$ with $H=\varliminf_{\text {m }} H_{\alpha}$. By construction we have injections $H_{\alpha} \rightarrow G_{\alpha}$. Set theoretically, we have $G / H=\varliminf G_{\alpha} / H_{\alpha}$. By Proposition (5), we can identify $G / H$ as a provariety as a subset of $\prod G_{\alpha} / H_{\alpha}$.

For use in our main theorem, we now investigate when the global sections of a provariety $G / H$ as in Theorem (4) separate the points of $G / H$. We have to allow here for the possibility (in positive characteristic) of bijective nonisomorphisms.

Theorem 5. Let $H$ be a pro-affine subgroup of the pro-algebraic group $G$. Then $K[G]^{H}$ separates the points of $G / H$ if and only if there exists a bijective morphism of provarieties from $G / H$ (with its natural provariety structure) into a provariety $W=\lim _{i} W_{i}$ where each $W_{i}$ is a quasi-affine algebraic variety.

Proof. We retain the notation of the proof of Theorem (4). Note that the inverse system $\left\{G_{\alpha} / H_{\alpha} \mid \alpha \in \mathcal{A}\right\}$ satisfies the transition conditions of Lemma (5). We let $K_{\alpha}$ denote the kernel of the surjection $p_{\alpha}$. 
If there is a provariety $W$ as in the theorem, then for a cofinal subset $\mathcal{A}^{\prime} \subseteq \mathcal{A}$, such that for all $\alpha \in \mathcal{A}^{\prime}$, there is an injective map $G_{\alpha} / H_{\alpha} \subset X_{\alpha}$ with $X_{\alpha}$ affine. We can replace $X_{\alpha}$ by the appropriate affine variety so that $\Gamma\left(X_{\alpha}, \mathcal{O}_{X_{\alpha}}\right)=K\left[G_{\alpha}\right]^{H_{\alpha}}=K[G]^{H K_{\alpha}}$; this makes $G_{\alpha} / H_{\alpha} \rightarrow X_{\alpha}$ a left $G$ equivariant embedding. By Theorem (4) we have an embedding $G / H \rightarrow \prod_{\mathcal{A}^{\prime}} G_{\alpha} / H_{\alpha}$, which we compose with the embedding $\prod_{\mathcal{A}^{\prime}} G_{\alpha} / H_{\alpha} \rightarrow \prod_{\mathcal{A}^{\prime}} X_{\alpha}$. It follows that the sections of the pro-affine variety $\prod X_{\alpha}$ separate the points of $G / H$, and hence that $\Gamma\left(G / H, \mathcal{O}_{G / H}\right)=K[G / H]=K[G]^{H}$ separates the points of $G / H$.

Conversely, suppose that $A=K[G]^{H}$ separates the points of $G / H$. Let $A_{\alpha}=K[G]^{H K_{\alpha}} \subseteq K\left[G_{\alpha}\right] . A_{\alpha}$ is finitely generated over $K$ and left $G$ stable. Let $X_{\alpha}=\operatorname{Alg}_{K}\left(A_{\alpha}, K\right)$ be the corresponding affine variety with global sections $A_{\alpha} . \quad G$, and $G_{\alpha}$, operate on the left on $X_{\alpha}$. The function $e: K[G] \rightarrow K$ determines, by restriction, a point $e_{\alpha} \in X_{\alpha}$. Let $H_{\alpha}$ be the stabilizer of $e_{\alpha}$ in $G_{\alpha}$ and $H(\alpha)$ the stabilizer of $e_{\alpha}$ in $G$, so that $H(\alpha)=p_{\alpha}^{-1}\left(H_{\alpha}\right)$ and $G_{\alpha} / H_{\alpha}=$ $G / H(\alpha)$. Then $G_{\alpha} / H_{\alpha}$ embeds in $X_{\alpha}$; we let $W_{\alpha}$ be its image; $W_{\alpha}$ is, of course, quasi-affine and the map $G_{\alpha} / H_{\alpha} \rightarrow W_{\alpha}$ is bijective. Note that $H \subseteq H(\alpha)$ for all $\alpha .\left(X_{\alpha}\right),(G / H(\alpha))$, and $\left(G_{\alpha} / H_{\alpha}\right)$ form inverse systems, and we have an injective morphism $\lim G_{\alpha} / H_{\alpha} \rightarrow \lim X_{\alpha}$ and an isomorphism $\lim G / H(\alpha) \rightarrow$ $\varliminf_{\infty} G_{\alpha} / H_{\alpha}$. Regard the limits as subsets of the corresponding products. $G$ acts on the products and preserves the inverse limits. We have $e_{\infty}=\left(e_{\alpha}\right) \in \prod X_{\alpha}$ belongs to $\varliminf_{\alpha} X_{\alpha}$. Let $H_{\infty}$ be the stabilizer of $e_{\infty}$, and note that $H_{\infty}=$

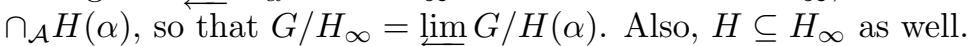

We have a canonical map $G / H_{\infty} \rightarrow G / H(\alpha)=G_{\alpha} / H_{\alpha}$ for each $\alpha$, hence a map (necessarily an injection) to $\prod G_{\alpha} / H_{\alpha}$ whose image is in $\varliminf_{\alpha} G_{\alpha} / H_{\alpha}$. Summarizing, we have maps

$$
G / H \rightarrow G / H_{\infty} \rightarrow \varliminf^{\lim } G_{\alpha} / H_{\alpha} \rightarrow \lim _{\alpha} X_{\alpha}
$$

and we consider the composite $\phi: G / H \rightarrow \lim X_{\alpha}$.

Let $X=\varliminf_{\lim _{\alpha}} X_{\alpha}$. By construction, $\Gamma\left(X, \mathcal{O}_{X}\right)=A$, and $X=\operatorname{Alg}_{K}(A, K)$, so that $A$ separates the points of $X$. Since $\phi^{*}\left(\Gamma, \mathcal{O}_{X}\right)=A=K[G]^{H} \subseteq$ $\Gamma\left(G / H, \mathcal{O}_{G / H}\right)$, and $K[G]^{H}$ separates the points of $G / H$, we conclude that $\phi$ is an injection. (If $\phi(a H)=\phi(b H)$, then $\forall f \in A, f(a H)=\phi^{*} f(a H)=$ $f(\phi(a H))=f(\phi(b H))=\phi^{*} f(b H)=f(b H)$, so $a H=b H$.) It then follows that $G / H \rightarrow G / H_{\infty}$ is an injection, so that $H=H_{\infty}$. It follows that

$$
G / H=G / H_{\infty}=\varliminf^{\lim } G / H(\alpha)=\varliminf_{\lim _{\alpha}} G_{\alpha} / H_{\alpha}
$$

and

$$
\varliminf_{\alpha} G_{\alpha} / H_{\alpha} \rightarrow W=\varliminf W_{\alpha}
$$

so that $G / H$ maps bijectively to $W$, as desired.

With Theorem (5) we have shown the equivalence of conditions (6) and (7) of our Main Theorem. Combined with the equivalences of conditions (1) through (6) shown in Theorem (3), this completes the proof of the Main Theorem. 


\section{References}

[1] Bialynici-Birula, A., Hochschild, G., and Mostow, G.D. Extensions of representations of algebraic groups, Amer. J. Math. 85 (1963) 131-144.

[2] Grosshans, F. Observable groups and Hilert's fourteenth problem, Amer. J. Math. 95 (1973) 229-253.

[3] Grosshans, F. Algebraic homogeneous spaces and invariant theory Lecture Notes in Mathematics 1673, Springer-Verlag, Berlin, 1997. vi+148 pp.

[4] Hochschild, G., and Mostow, G.D. Representations and representative functions of Lie groups, Ann. of Math. 66 (1957) 495-542.

[5] Hochschild, G., and Mostow, G.D. Pro-affine algebraic groups, Amer. J. Math. 91 (1969) 1127-1140.

[6] Hochschild, G., and Mostow, G.D. Complex analytic groups and Hopf algebras, Amer. J. Math. 91 (1969) 1141-1151.

[7] Kovacic, J. Pro-algebraic groups and the Galois theory of differential fields, Amer. J. Math. 95 (1973) 507-536.

[8] Kowalski, P. and Pillay, A., J. Pro-algebraic and differential algebraic group structures on affine spaces, Amer. J. Math. 122 (2000) 213-221.

[9] Lee, Dong Hoon; Wu, Ta Sun. On observable subgroups of complex analytic groups, J. Algebra 173 (1995) 166-179.

[10] Leptin, H. Linear kompakte modulun und ringe, Math. Z. 62 (1955), 241267.

[11] Lubotzky, A. and Magid, A., Cohomology of unipotent and prounipotent groups J. Algebra 74 (1982) 76-95.

[12] Peterson, B. Extensions of pro-affine algebraic groups, Pacific J. Math. 77 (1978) 189-231.

[13] Sukhanov, A. A. The description of observable subgroups of linear algebraic groups, (Russian) Mat. Sb. (N.S.) 137(179) (1988) 90-102, 144; translation in Math. USSR-Sb. 65 (1990) 97-108.

[14] Waterhouse, W. Introduction to Affine Group Schemes, Graduate Texts in Mathematics 66, Springer-Verlag, New York, 1979. 